\title{
COMPOSIÇÃO QUÍMICA E PERCENTUAL DE ADEQUAÇÃO DA DIETA DOS SERVIDORES DO INSTITUTO NACIONAL DE PESQUISAS DA AMAZÔNIA, MANAUS, AM, BRASIL.
}

\author{
Dionisia NAGAHAMA'; Lúcia K. O. YUYAMA'; Jaime P. L. AGUIAR'; Sonja H. M. \\ MACEDO $^{2}$; Lina YONEKURA ${ }^{2}$; Fernando H. ALENCAR'; Déborah I. T. FÁVARO ${ }^{3}$; \\ Claudia AFONSO ${ }^{3}$; Marina B. A. VASCONCELOS ${ }^{3}$
}

RESUMO - Foi avaliada a composição quimica e percentual de adequação das dietas coletadas por meio da porção em duplicata de 36 servidores do Instituto Nacional de Pesquisas da Amazônia (INPA). De acordo com os resultados obtidos, verificou-se uma diversificação de alimentos freqüentemente consumidos, dentre eles: açúcar $(91,7 \%)$, arroz $(80,6 \%)$, café $(77,8 \%)$, leite $(72,3 \%)$, carne bovina e pão $(63,9 \%)$, farinha de mandioca $(58,4 \%)$, batata inglesa $(55,6 \%)$, feijão $(50,04 \%)$, aves $(38,9 \%)$, banana, cenoura e embutidos $(33,4 \%)$, ovo, refrigerantes e tomate $(30,6 \%)$. Considerando as recomendações nutricionais para a faixa etária de 25 a 50 anos, as adequações calóricas foram $40 \%$ e $52,7 \%$ para homens e mulheres respectivamente, protéica $76,4 \%$ (homens) e $96,3 \%$ (mulheres). Os minerais $\mathrm{Ca}, \mathrm{Mg}$ e $\mathrm{Zn}$ (homens) apresentaram adequação inferior a $50 \%$, enquanto que o Fe apresentou adequaçào de $89,1 \%$ (homens) e $59.4 \%$ (mulheres). Os minerais $\mathrm{Na}, \mathrm{Cu}, \mathrm{Cl}, \mathrm{Cr}$ e I apresentaram valores acima do recomendado em ambos os sexos. Apesar da grande variedade de alimentos ingeridos por esta população, verificou-se valores limitantes para a maioria dos nutrientes, independentemente do sexo. Mesmo pertencendo a um nivel sóciocultural mais privilegiado, fica patente a importância da orientação alimentar a esse grupo populacional, pois é um dos pontos principais no que concerne as condições de saúde e nutrição da população.

Palavras-chave: dieta, adulto, composição quimica, avaliação nutricional.

Chemical Composition and Adequacy Percentile of the Servers Feeding of the National Institute of Amazonia Research, Manaus, Am., Brazil.

\begin{abstract}
The chemical composition of the duplicate food collections obtained from 36 servers of the National Institute of Researches of Amazônia (INPA) was evaluated and as percentage of recommended intake. The most frequently food consumed they were: sugar $(91.7 \%)$, rice $(80.6 \%)$, coffee $(77.8 \%)$, milk $(72.3 \%)$, bovine meat and bread $(63.9 \%)$, cassava flour $(58.4 \%)$, potato $(55.6 \%)$, bean $(50.0 \%)$, birds $(38.9 \%)$, banana, carrot and sausages $(33.4 \%)$, egg, sodas and tomato $(30.6 \%)$. The results obtained showed the caloric deficiency of $40 \%$ and $52,7 \%$ respectively for adult men and adult women (25 to 50 years), protein of $76,4 \%$ (men) and $96,3 \%$ (women). The minerals $\mathrm{Ca}, \mathrm{Mg}$ and $\mathrm{Zn}$ (men) were deficient whit an adequacy of about $50 \%$. When compared with the recommended intakes, Fe was deficient for the adult women $(59.4 \%)$. The minerals $\mathrm{Na}, \mathrm{Cu}, \mathrm{Cl}, \mathrm{Cr}$ and I values were very high. Although of the great variety of foods ingested by this population, it was verified values are near the lowest limited set by RDA for most of the nutrients. Same belonging at a more privileged socio-economic living conditions, it is patent the importance of the alimentary orientation to that population group, because it is one of the main points in what it concerns the conditions of health and nutrition of the population.
\end{abstract}

Key-words: diet, adult, chemical composition, nutritional assessment.

${ }^{1}$ Coordenação de Pesquisas em Ciências da Saúde - INPA - Caixa postal 478 - 69011-970Manaus, AM.

${ }^{2}$ Programa Institucional de Bolsa de Iniciação Cientifica - INPA

${ }^{3}$ Supervisão de Radioquímica IPEN/ CNEN - SP. 


\section{Introdução}

Dados sobre a ingestão alimentar são necessários para predizer a adequação de suprimentos alimentares, monitoração de tendências na utilização de alimentos, estimar exposição aos contaminantes, e, identificação de grupos populacionais de alto risco (Buzzard, 1994).

Grande parte da população dos países do terceiro mundo, vive em situação de penúria, e, miséria, onde a desnutrição é uma constante, por falta de acesso a uma alimentação adequada. Não obstante, a obesidade, e, as doenças crônico-degenerativas como o câncer, osteoporose, cardiovasculares e diabetes mellitus, assumem a magnitude de um problema de saúde pública, nas populações mais privilegiadas, demonstrando a ocorrência de desnutrição, por excesso e dieta desbalanceada (Dutra Oliveira et al., 1996).

$\mathrm{O}$ método de inquérito alimentar recordatório de 24 horas, é o comumente utilizado para se obter o padrão alimentar de grupos populacionais, sendo um instrumento essencial na avaliação perfil alimentar (Fujimori et al., 1986).

$\mathrm{Na}$ região Amazônica, alguns estudos, tem se reportado a esse método, no sentido de se conhecer o padrão alimentar e prováveis deficiências de nutrientes que poderiam comprometer o estado nutricional da população (Shrimpton et al., 1983, Lehti,1989; Nagahama et al., 1990). Entretanto, as análises são baseadas em tabelas de composição de alimentos, e sabe-se que a informação obtida por esse método não proporciona dados reais sobre o consumo de nutrientes, principalmente quanto aos teores de micronutrientes, tendo sido recomendada a análise quimica laboratorial da dieta por meio da porção em duplicata (Abdulla et al, 1979 ). Somam-se ainda, a dificuldade em se conseguir informações sobre a composição de micronutrientes de alimentos típicos da região amazônica, motivo pelo qual estamos utilizando o método da porção em duplicata (Yuyama et al.,1992, Nagahama, 1994, Yuyama \& Cozzolino,1995, Yuyama et al., 1999).

Considerando os poucos dados disponiveis sobre o padrão alimentar da população adulta com um nivel sócioeconômico e cultural mais privilegiado, avaliou-se o perfil alimentar e a adequação dos macro e micronutrientes da dieta dos servidores do Instituto Nacional de Pesquisas da Amazônia.

\section{Material e Métodos}

Foram avaliadas uma sub amostra de 36 dietas coletadas por meio de porção em duplicata, consumida em um dia da semana. Os servidores foram orientados a coletar todos os alimentos, preparações e liquidos consumidos no lar ou extra lar até a ultima refeição do dia. Os alimentos consumidos ao longo do dia, foram acondicionados em sacos plásticos e mantidos em geladeira até o momento da entrega no laboratório de Nutrição, da Coordenação de 
Pesquisas em Ciência da Saúde do Instituto Nacional de Pesquisas da Amazônia, e, posteriormente congelados até a análise.

As dietas foram secas em estufas ventiladas a $60^{\circ} \mathrm{C}$ até peso constante, para a determinação do teor de umidade, pulverizadas e homogeneizadas para análise quimica. Uma alíquota foi enviada à Instituição de Pesquisas Energéticas e Nucleares-Comissão Nacional de Energia Nuclear (IPEN/CNEN), para análises de elementos minerais. A determinação da composição química foi realizada, no Laboratório de Nutrição e Físico-Química de Alimentos do INPA, de acordo com as normas da AOAC (1995). Para a determinação do teor de fibra alimentar solúvel e insolúvel seguiuse o método de Asp et al. (1983). A análise de minerais de ativação com nêutrons, conforme descrito no trabalho de Yuyama et al. (1997). Com o material de referência utilizouse certificado Peach Leaves (NISTSRM 1547), de acordo com as recomendações de Cornelis (1992).

$A$ adequação nutricional da dieta seguiu as recomendações da National Academy Science/National Research Council- (NAS/NRC, 1989), tendo como referencial adultos masculino e feminino na faixa etária de 25 a 50 anos.

\section{Resultados e Discussão}

Dos servidores que participaram do estudo, verificou-se que a idade média foi de $38 \pm 9,5$ anos, com uma variação de 19 a 61 anos, sendo que a maioria $(66,7 \%)$ encontrava-se na faixa etária de 30 a 49 anos, e $(61,1 \%)$ do sexo feminino. Aproximadamente a metade $(55,9 \%)$ tinha nivel superior, sendo técnicos $(27,8 \%)$, pesquisadores e de gestão $(25 \%)$. Quanto a procedência, o destaque foi para a região Norte com $(68,6 \%)$ e região Sudeste $14,2 \%$ (Tab. 1).

Analisando o consumo alimentar dos servidores, observou-se que dentre os grupos de alimentos: Cereais e Tubérculos contribuíram com um maior percentual na dieta $(27 \%)$, seguidos das Carnes e Ovos (16\%) e Gorduras e Doces (12\%) (Fig. 1).

Verificou-se que os alimentos mais freqüentemente consumidos pelos servidores foram: açúcar $(91,7 \%)$, arroz $(80,6 \%)$, café $(77,8 \%)$, leite $(72,3 \%)$, carne bovina e pão $(63,9 \%)$, farinha de mandioca $(58,4 \%)$, batata $(55,6 \%)$, feijão $(50,0 \%)$, aves $(38,9 \%)$, banana, cenoura e embutidos $(33,4 \%)$, ovo, refrigerantes e tomate (30,6\%), (Tab. 2).

Vale ressaltar que o peixe, alimento tipicamente regional, foi o menos consumido dentro do grupo das carnes, diferentemente, de outros estudos da região enfocando a população adulta (Shrimpton \& Giugliano, 1979; Giugliano et al., 1981; 1984, Yuyama et al., 1989).

A composição química da dieta dos 36 servidores está demonstrado na (Tab. 3).

O valor calórico de 1160,1 Cal. (Tab. 5), evidencia um provimento deficitário para os adultos de ambos os sexos considerando o referencial de 25 a 50 anos, segundo as recomendações do RDA (1989). Sendo assim, este provimento calórico atende as 
Tabela 1. Características dos 36 servidores do Instituto Nacional de Pesquisas da Amazônia.

\begin{tabular}{|c|c|c|c|}
\hline CARACTERISTICAS & HOMENS $N=14(38,9 \%)$ & MULHERES $N=22(61,1 \%)$ & TOTALN $=36(100 \%)$ \\
\hline Idade média & $40+10,7$ & $37+8,8$ & $38+9,5$ \\
\hline \multicolumn{4}{|l|}{ Faixa etária } \\
\hline 18 a 29 & $3(21,4 \%)$ & $5(22,7 \%)$ & $8(22,2 \%)$ \\
\hline 30 a 49 & $9(64,3 \%)$ & $15(68,2 \%)$ & $24(66,7 \%)$ \\
\hline 50 a 69 & $2(14,3 \%)$ & $2(9,1 \%)$ & $4(11,1 \%)$ \\
\hline \multicolumn{4}{|l|}{ Escolaridade $(\mathrm{N}=34)$} \\
\hline 0 & 0 & $1(4,5 \%)$ & $1(2,9 \%)$ \\
\hline $1^{\circ}$ & $1(8,3 \%)$ & $2(9,1 \%)$ & $3(8,8 \%)$ \\
\hline $2^{\circ}$ & $4(33,3 \%)$ & $7(31,8 \%)$ & $11(32,4 \%)$ \\
\hline $3^{\circ}$ & $7(58,3 \%)$ & $12(54,5 \%)$ & $19(55,9 \%)$ \\
\hline \multicolumn{4}{|l|}{ Funçăo } \\
\hline Pesquisador & $3(21,4 \%)$ & $6(27,3 \%)$ & $9(25 \%)$ \\
\hline Técnico & $6(42,9 \%)$ & $4(18,2 \%)$ & $10(27,8 \%)$ \\
\hline Estudante & $3(21,4 \%)$ & $4(18,2 \%)$ & $7(19,4 \%)$ \\
\hline Gestão & $2(14,3 \%)$ & $7(31,8 \%)$ & $9(25 \%)$ \\
\hline Conservadora & 0 & $1(4,5 \%)$ & $1(2,8 \%)$ \\
\hline \multicolumn{4}{|l|}{ Procedência $(\mathrm{N}=35)$} \\
\hline Norte & $7(53,8 \%)$ & $17(77,3 \%)$ & $24(68,6 \%)$ \\
\hline Nordeste & $1(7,7 \%)$ & $2(9,1 \%)$ & $3(8,6 \%)$ \\
\hline Sudeste & $2(15,4 \%)$ & $3(13,6 \%)$ & $5(14,2 \%)$ \\
\hline Estrangeira & $3(23,1 \%)$ & 0 & $3(8,6 \%)$ \\
\hline
\end{tabular}

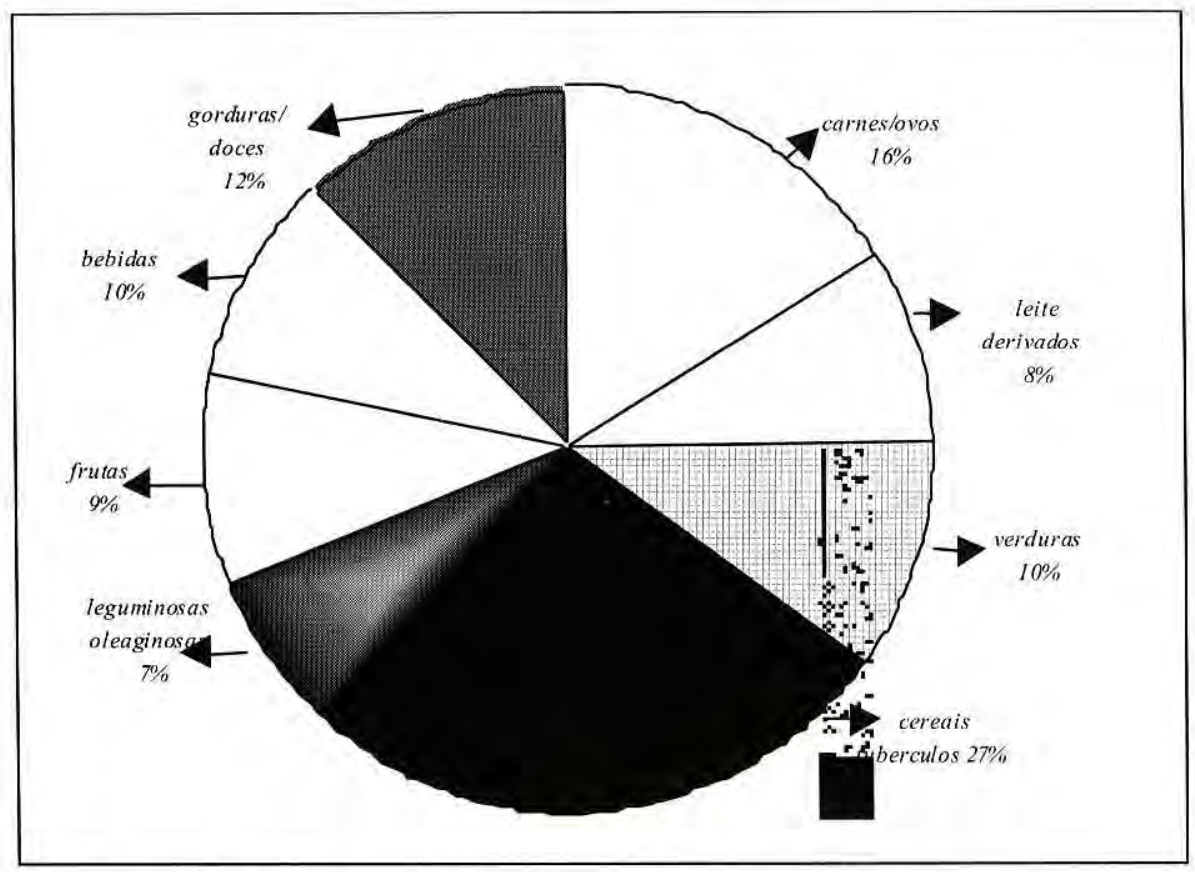

Figura 1. Distribuição da freqüência do consumo por grupos de alimentos. 
Tabela 2. Alimentos freqüentemente consumidos pelos servidores.

\begin{tabular}{|c|c|c|c|}
\hline CARNES E OVOS & FREQÜÊNCIA(\%) & VERDURAS & FREQÜÊNCIA(\%) \\
\hline Carne bovina & 63,9 & Cenoura & 33,4 \\
\hline Aves & 38,9 & $\sqrt{1}$ & 30,6 \\
\hline Enlatados/embutidos & 33,4 & Alface & 13,9 \\
\hline Ovo & 30,6 & pepino & 27,8 \\
\hline Peixe & 16,7 & Repolho & 8,3 \\
\hline Outros & 19,5 & Outros & 16,8 \\
\hline LEITE E DERIVADOS & & LEGUMINOSAS OLEAGINOSAS & \\
\hline Leite & 72,3 & Feijão & 50,0 \\
\hline Queijo & 27,8 & Ervilha & 11,1 \\
\hline logurte & 5,6 & Azeitona & 11,1 \\
\hline FRUTAS & & Feijão verde & 8,3 \\
\hline Banana & 33,4 & Outros & 5,6 \\
\hline Mamão & 11,1 & GORDURAS E DOCES & \\
\hline Laranja & 8,3 & Açúcar & 91,7 \\
\hline Abacaxi & 8,3 & Margarina & 25,0 \\
\hline Maracujá & 8,3 & Geleia/doces & 11,1 \\
\hline Maçã & 8,3 & Chocolate & 8,3 \\
\hline \multirow[t]{2}{*}{ Outros } & 16,7 & Bolo & 8,3 \\
\hline & & Outros & 8,3 \\
\hline BEBIDAS & & CEREAIS E TUBÉRCULOS & \\
\hline Café & 77,8 & pão & 63,9 \\
\hline Refrigerante & 30,6 & biscoito & 16,7 \\
\hline \multirow[t]{5}{*}{ Chá } & 11,1 & arroz & 80,6 \\
\hline & & farinha de mandioca & 58,4 \\
\hline & & farinha de tapioca & 22,2 \\
\hline & & batata & 55,6 \\
\hline & & Outros & 5,6 \\
\hline
\end{tabular}

mulheres em $52,7 \%$ das necessidades, enquanto que para os homens somente em $40,0 \%$.

Valores inferiores em calorias foram observados em outras dietas brasileiras (Cozzolino,1992; Harada, 1993, Boaventura, 1994, Pedrosa, \&
Cozzolino,1990), e, regionais (Yuyama et al., 1992; Nagahama, 1994). Quanto a distribuição calórica da dieta analisada, pode-se caracterizá-la como hiperproteica, normolipídica e normoglicídica (Tab. 4), diferentemente, das dietas regionais consumidas pela 
Tabela 3. Composição quimica da dieta* dos servidores do INPA.

\begin{tabular}{cccccccc}
\hline Umidade $(\%)$ & Proteina $(\mathrm{g})$ & Lipidios $(\mathrm{g})$ & Glicidios $(\mathrm{g})$ & Cinzas $(\mathrm{g})$ & Fibrainsolúvel(g) & Fibrasolúvel(g) & Energia(Cal) \\
$\mathbf{7 2 , 9} 9 \pm 7,5$ & $\mathbf{4 , 5 \pm 0 , 0 0 1}$ & $2,6 \pm 0,02$ & $\mathbf{1 7 , 8}$ & $0,9 \pm 0,003$ & 1,3 & 0,0 & 111,7 \\
\hline
\end{tabular}

"Em $100 \mathrm{~g}$ da dieta.

Tabela 4. Distribuição calórica dos nutrientes .

\begin{tabular}{ccc}
\hline Nutrientes & Dieta $(\%)$ & Recomendação* \\
\hline Proteina & 16,1 & $10-15$ \\
Lipidio & 20,3 & $20-25$ \\
Glicídio & 63,6 & $50-60$ \\
\hline
\end{tabular}

*Vannucchi et al.,1990

Tabela 5. Composição e adequação da dieta dos servidores do Instituto Nacional de Pesquisas da Amazônia.

\begin{tabular}{|c|c|c|c|c|c|}
\hline \multirow[t]{2}{*}{ Nutrientes } & \multirow{2}{*}{$\begin{array}{l}\text { Ingestão } \\
\text { média } \\
\text { diária }\end{array}$} & \multicolumn{2}{|c|}{ Recomendação. } & \multicolumn{2}{|c|}{ Adequação $(\%)$ * } \\
\hline & & $M$ & $\mathrm{~F}$ & M & $F$ \\
\hline Proteína $(\mathrm{g})$ & 41,7 & 63 & 50 & 66,2 & 83,4 \\
\hline Calorias (Cal) & 1034,0 & 2900 & 2200 & 35.7 & 47,0 \\
\hline $\mathrm{Na}(\mathrm{mg})$ & 2813,84 & 500 & 500 & 562,8 & 562,8 \\
\hline $\mathrm{K}(\mathrm{mg})$ & 1219,73 & 2000 & 2000 & 61,0 & 61,0 \\
\hline $\mathrm{Ca}(\mathrm{mg})$ & 285,33 & 800 & 800 & 35,7 & 35.7 \\
\hline $\mathrm{Mg}(\mathrm{mg})$ & 105,49 & 350 & 280 & 30.1 & 37,7 \\
\hline $\mathrm{Cu}(\mathrm{mg})$ & 6,68 & $1,5-3,0$ & $1,5 \cdot 3,0$ & $445,3-222,7$ & $445,3-222,7$ \\
\hline $\mathrm{Fe}(\mathrm{mg})$ & 8,91 & 10 & 15 & 89.1 & 59,4 \\
\hline$M n(m g)$ & 1,95 & $2,0-5,0$ & $2,0-5,0$ & $97,5-39$ & $97.5-39$ \\
\hline $\mathrm{Cl}(\mathrm{mg})$ & 3712,9 & 750 & 750 & 495,1 & 495,1 \\
\hline $\mathrm{Zn}(\mathrm{mg})$ & 6,54 & 15,0 & 12,0 & 43,6 & 54,5 \\
\hline $\mathrm{Cr}(\mu \mathrm{g})$ & 222,68 & $50-200$ & $50-200$ & $445,4-111,3$ & $445,4-111,3$ \\
\hline$S e(\mu g)$ & 35,63 & 70 & 55 & 50,9 & 64,8 \\
\hline$I(\mu g)$ & 580 & 150 & 150 & 386,6 & 386,6 \\
\hline Fibra (g) & 11,8 & 20 & 20 & 59 & 59 \\
\hline
\end{tabular}

* de acordo com a RDA (1989)

** quantidade média da dieta: $922,2 \mathrm{~g}$.

população de baixa renda, onde predominam a oferta de glicídios, como fonte energética, já nas dietas dos estudantes universitários, as proteinas e os lipídios, tiveram uma maior contribuição (Harada,1993; Boaventura, 1994).

A deficiência de energia na alimentação representa um grave problema nutricional, pois se a ingestão 
calórica não satisfaz as necessidades, torna-se muito difícil manter o aproveitamento adequado dos demais nutrientes essenciais (Tudisco et al.,1985).

Apesar da grande freqüência de ingestão de alimentos de origem animal, fontes de proteínas de alto valor biológico, a adequação deste nutriente foi de $76,4 \%$ para o sexo masculino e 96,3 para o sexo feminino, resultados inferiores aos habitualmente encontrados em outros grupos populacionais, onde geralmente o aporte protéico está bem acima do recomendado, conforme demonstrado em vários estudos (Cozzolino, 1992, Harada, 1993; Boaventura, 1994; Yuyama et al., 1992; Nagahama, 1994).

Além do resultado deficitário de proteína, outro fator que poderia comprometer a sua utilização metabólica seria o baixo valor calórico onde, provavelmente, acarretaria o desvio metabólico da proteína ingerida para o suprimento das necessidades energéticas existentes, com o prejuízo da finalidade principal deste nutriente, ou seja a sua função plástica.

O consumo de fibra alimentar também foi baixo, com uma adequação por volta de $50 \%$. Apesar da variedade de alimentos consumidos, ressalta-se a necessidade da presença de alimentos ricos em fibras, em especial os folhosos e frutos, considerando a relevância dos mesmos na nutrição humana.

Em relação aos minerais, a concentração de cálcio, magnésio, zinco e ferro (mulheres) não satisfaz as necessidades diárias dos indivíduos adultos em referência, provavelmente, pela baixa ingestão calórica, e, de fontes alimentares capaz de atender as exigências nutricionais dos elementos acima citados. As concentrações desses elementos minerais, foram inferiores aos encontrados nos estudos regionais (Yuyama et al., 1992, Nagahama,1994; Yuyama \& Cozzolino,1995).

Sódio, cobre, cromo e iodo apresentaram indices superiores ao recomendado, e, manganês valores satisfatórios em relação ao mínimo necessário.

Os nutrientes mais deficientes na dieta analisada foram o magnésio (homens), cálcio, zinco, selênio, potássio e ferro, notadamente em mulheres.

$\mathrm{O}$ cálcio foi um dos nutrientes que apresentou o menor índice de adequação, geralmente esta deficiência é uma característica das dietas brasileiras (Cozzolino, 1992; Pedrosa \& Cozzolino, 1990; Yuyama et al, 1995), entretanto muito inferior ao encontrado em outros estudos (Boaventura, 1994; Harada, 1993), que analisaram um grupo populacional de nível sócio-econômico mais próximo ao nosso grupo.

Em relação ao zinco, é freqüente o registro de valores deste mineral entre 11 a $13 \mathrm{mg} / \mathrm{dia}$ (Moser-Veillon, 1990), entretanto, o resultado obtido na dieta dos servidores do INPA $(6,54 \mathrm{mg})$ foi expressivamente inferior aquele referido para a dieta de Manaus (10,68 mg), (Yuyama \& Cozzolino, 1995). A literatura refere que no aproveitamento do zinco, devem ser considerados os 
fatores que podem afetar a sua biodisponibilidade, tais como: a presença do cálcio, ferro e fibra, o que não se aplica ao presente estudo, visto que nenhum destes nutrientes atingiu um nível de inadequação, que pudesse comprometer este aproveitamento. O zinco é um microelemento, considerado essencial em diversas funções do organismo, incluindo crescimento e desenvolvimento, reprodução, função imune e sensorial, proteção antioxidante, estabilidade e integridade de membranas celulares (OMS, 1998).

$\mathrm{Na}$ dieta analisada, o ferro foi outro nutriente que apresentou um déficit de $59 \%$ em relação as necessidades para mulheres. A deficiência nutricional deste mineral é comum na Região Amazônica, principalmente em crianças e gestantes (Giugliano et al., 1978; Duarte, 1989; Yuyama et al, 1992). É importante salientar que o tipo da dieta, a origem do ferro e a presença de alguns fatores favorecem ou inibem a absorção desse nutriente. $\mathrm{O}$ ferro dietético, proveniente das carnes, é mais biodisponível que o das fontes de origem vegetal, este último, é melhor absorvido quando ingerido simultaneamente ao de origem animal (Fujimori, 1986). Do mesmo modo, a ingestão concomitante com o ácido ascórbico aumenta a biodisponibilidade do ferro dietético, favorecendo a sua absorção. Dentre os fatores inibidores, destacam-se: ácido fítico, polifenóis, cafeína e interação minerais, minerais (Yuyama \& Cozzolino,1992; Cozzolino,1997).

$\mathrm{O}$ teor de selênio encontrado na dieta dos servidores (35.63mg) não difere de outros estudos (Boaventura,1994; Cozzolino, 1992); entretanto foi muito inferior ao resultado da dieta de Manaus, e, de outras regiões como Santa Catarina e Mato Grosso (Fávaro et al., 1997). Um dos fatores determinantes dessas diferenças, pode ser devido as características dos solos e localização geográfica. $\mathrm{Na}$ região Amazônica existem solos de terra firme, considerados pobres em nutrientes e solo de várzea extremamente rico, o que demonstra a importância de se determinar os teores destes micronutrientes em alimentos ou dietas. Entretanto, ainda é questionável as reais necessidades nutricionais desses minerais para a população humana (Yuyama \& Cozzolino, 1995).

Vale ressaltar que a maioria dos produtos alimentícios é proveniente de outros Estados, principalmente do Sudeste, o que possivelmente tenha contribuido no resultado encontrado para o Se, no presente estudo.

Em relação ao sódio, sua concentração foi superior a recomendada, e, semelhante aos resultados referidos em outros estudos (Holbrook et al., 1984; Eaton et al., 1984; Nagahama, 1994). Geralmente a ingestão deste mineral ultrapassa, em muito, as recomendações. Segundo alguns levantamentos de Carraza (1988), os valores variam desde $1,75 \mathrm{~g}$ a $2.75 \mathrm{~g}$ de sódio por dia (EUA), até $11,1 \mathrm{~g}$ a 14, 6 g/dia (Japão).

$\mathrm{O}$ teor de $\mathrm{Cu}$ na dieta, também foi muito elevado, diferindo de outros estudos que apresentaram inferiores ao recomendado (Harada,1993; Fávaro et al., 1997).

Os resultados dos demais 
elementos minerais ( $\mathrm{K}, \mathrm{Mn}, \mathrm{Cl}, \mathrm{I})$ são citados para efeito de registro para futuras comparações, pois são poucos os estudos referentes a estas análises (Yuyama \& Cozzolino, 1995; Fávaro et al., 1997).

\section{CONCLUSÃO}

A dieta consumida pelos integrantes do presente estudo, não atende, em sua plenitude, as recomendações nutricionais, ficando implícito a necessidade de uma reorientação alimentar, apesar do seu poder aquisitivo e padrão sócio-cultural.

\section{Agradecimentos}

Ao Instituto Nacional de Pesquisas da Amazônia, a todos os servidores, estagiários, bolsistas e estudantes do Instituto que voluntariamente participaram da pesquisa.

\section{Bibliografia citada}

Abdulla, M.; Anderson, I.; Belfrage, P.; Dencker, I.; Jagerstad, M.; Melander, A.; Norden, A.; Schersten, B.; Thulin, T.; Akesson, B. 1979. Assesment of food consumption. Scand. J. Gastroenterology, Oslo, 14 (52):28-42.

Asp, N.G.; Johansson, C.G.; Hallmer, H.; Siljestrom, M. 1983. Rapid enzymatic assay of insoluble end soluble dietary fiber. J. Agric. Food. Chem., Washington, 31: 476-482.

Association Of Official Analytical Chemists/ AOAC. 1990. Official methods of analyses. 15 ed., Arlinton.

Boaventura, G.T. 1994. Avaliação nutricional relativa ao selênio de estudantes da Faculdade de Ciências Farmacêuticas da USP. São Paulo. Tese de Doutorado da Faculdade de Ciências Farmacêuticas USP. $117 \mathrm{p}$.
Buzzard,I.M. \& Silvert, Y.A. 1994. Research priorities and recommendations for dietary assessment methodology. Am. J. Clin. Nutr.,Betheda, 59: 2755-2805

Carraza, F.R. 1988. Minerais em dietas latinoamericanas. Arch. Latinoamer. Nutr., 38 (3):599-42.

Cornelis,R. 1992. Use of references materials in trace element analysis of foodstuffs. Food Chem. Barking,43: 307-13.

Cozzolino, S.M.F. 1992, Biodisponibilidade de zinco em dieta. Estudo com ratos em diferentes fases do desenvolvimento. Tese de Livre-Docência da Faculdade de Ciências Farmacêuticas - USP., São Paulo. 118p.

Duarte, M.R.B. 1989. Estudos dos fatores de risco nutricional em crianças matriculadas em pré-escolar das redes de ensino particular, estadual e municipal da cidade de Manaus (AM) em 1987. Avaliação dos inquéritos alimentar, sócio-econômico $e$ cultural. Manaus-AM. Dissertação de mestrado, Instituto Nacional de Pesquisas da Amazônia/Fundação Universidade do Amazonas. $114 \mathrm{p}$.

Dutra de Oliveira, J. E.; Carvalho da Cunha, F. C.; Marchini, J. S.. 1996. A desnutrição dos Pobres e dos Ricos, Ed. Sarvier, São Paulo-SP, Brasil.

Eaton, P.M.; Wharton, P.A.; Wharton, B.A. 1984. Nutrient intake of pregnant Asian women at Sorrento Maternity Hospital, Birmingham. Br. J. Nutr. 52 (2): 457-68.

Fávaro, D. I. D.; Hui, M. L.T.; Cozzolino, S. M. F.; Maihara, M. J. A.; Boaventura and Fujimori, E.; Shima, H.; Salum, M.S.L. 1986. Consumo alimentar de estudantes universitários do sexo feminino. Rev. Esc. Enf. USP, São Paulo, 20 (2):115-124.

Giugliano,R;; Giugliano,L.G.; Shrimpton, R. 1981.Estudos nutricionais da populaçōes rurais da Amazônia. I. Vàrzea do rio Solimões. Acta Amazonica, 11 (4):773-788.

Giugliano, R..; Shrimpton, R.; Marinho, H.A.; Giugliano,L.G. 1984. Estudos nutricionais das populações rurais da Amazônia. II. Rio Negro. Acta Amazonica, 14 (3-4):427-449.

Harada, L.M. 1993. Efeito da cimetidina na 
biodisponibilidade de zinco e cobre em dieta. Estudo em ratos. Tese de Mestrado da Faculdade de Ciências Farmacêuticas USP., São Paulo. 167p.

Holbrook, J.T.; Cottrell, S.L.; Smith Jr.; J.C. 1984. Correlations of changes in dietary potassium and sodium with blood pressure during a one-year study. Am. J. Clin. Nutr., 40 (suppl. 6) : 1390-92.

Kim, W.W.;Mertz, W.; Judd, J.T.; Marshall, M.W.; Kelsay, J.L.; Prather, E.S. 1984. Effect of making duplicate food collections on nutrient intakes calculated from diet records. Am. J. Clin. Nutr., Bethesda, 40: 1333-37.

Lehti, K.K. 1989. Iron, folic acid and zinc intakes and status of low socio-economic pregnant and lactating Amazonian women. European Journal of Clinical Nutrition, 43: 505-513.

Moser-Veillon, P.B. 1990. Zinc: consumption patterns and dietary recommendations. $J$. Am. Diet. Assoc, Chicago, 90: 1089-93.

Nagahama, D.; Marinho, H.A.;Rocha, Y.R.; Ferraroni, M.J.R.; Silva, N.B.; Castro, J. S.; Onety,J.A. 1990. Avaliação nutricional e alimentar de pré-escolares de uma creche de Manaus e a influência da entidade no estado nutricional de sua população. Acta Amazonica, 20:119-129

Nagahama, D. 1994. Estudo comparativo da composição quimica das 20 dietas representativas da ingestão dietética de mulheres em idade fértil de um bairro pobre de Manaus- AM. Manaus, AM. . Dissertação de Mestrado, Instituto Nacional de Pesquisas da Amazônia/ Fundação Universidade do Amazonas.80p.

National Academy Of Science/National Research Council. 1989. Recommended Dietary Allowances. 10 ed., Washington. $284 \mathrm{p}$.

OMS. 1998. Elementos traços na nutrição e saúde humanas. ROCA, São Paulo. 297 p.

Pedrosa, L.F.C. \& Cozzolino, S.M.F. 1990. Biodisponibilidade de zinco em dieta regional do nordeste. Rev. Farm. Bioq. Univ. S. Paulo, S.Paulo, 26:123-133.

Shrimpton, R. \& Giugliano, R. 1979. Consumo de alimentos e alguns nutrientes em Manaus, Amazonas. Acta Amazônica, 9 (1): 117-41.

Shrimpton, R.; Marinho, H.A.; Rocha, Y.S.; Alencar, F.H. 1983. Zinc supplementation in urban Amazonian mothers: concentrations of zinc and retinol in maternal serum and milk. Proc. Nutr. Soc., 42 (3): $122 \mathrm{~A}$.

Yuyama, L.K.O.; Rocha, Y.R.; Cozzolino, S.M. 1992. Composição quimica e percentual de adequação da dieta regional de ManausAM. Acta Amazonica, 22 (4):587-593.

Yuyama, L.K.O. \& Cozzolino, S.M.F. 1995. Determinação dos teores de $\mathrm{Zn}, \mathrm{Fe}, \mathrm{Ca}$, $\mathrm{Se}, \mathrm{Cu}, \mathrm{K}, \mathrm{Mg}$ e $\mathrm{Mn}$ na dieta regional de Manaus, AM. Rev. Inst. Adolfo Lutz, 55 (1):45-50.

Yuyama, L.K.O.; Aguiar, J.P.L.; Macedo, S.H.M.,;Gioia, T.; Yuyama, K.; Fávaro, D.I.T.; Afonso, C.; Vasconcellos, M.B.A.; Cozzolino, S.M.F. 1997. Determinação dos teores de elementos minerais em alimentos): ( convencionais e nào convencionais da região amazônica pela técnica de análise por ativação com nêutrons instrumental. Acta Amazonica,.27 (3):183-196.

Yuyama, L.K.O.; Vasquez, A.L.V.; Aguiar, J.P.L.; Macedo, S.H.M.,; Yonekura, L.; Nagahama, D.; Fonseca, C.W. 1999. Composição Química e adequação da Alimentação Oferecida aos pré-escolaresde uma Instiruição Beneficiente de Manaus , Amazonas, Brasil. Acta Amazonica, 29(4): 549-554.

Aceito para publicação em 22/05/2002 